ISSN: 2602-8085

Vol. 4, N 4.2, p. 48-64, abril - junio, 2020

\title{
Estrategias financieras para la sostenibilidad y el crecimiento del banco internacional agencia Riobamba período 2019 - 2021
}

\section{Financial strategies for the sustainability and growth of banco internacional agency Riobamba period 2019 - 2021}

Victor Oswaldo Cevallos Vique. ${ }^{1}$, Hernán Octavio Arellano Diaz. ${ }^{2}$, Glenda Marisol Santillán Valle. ${ }^{3} \&$ Paulina Elizabeth Valverde Aguirre. ${ }^{4}$

\begin{abstract}
.
DOI: $\underline{\text { https://doi.org/10.33262/cienciadigital.v4i2.1196 }}$

This research was carried out at the Banco Internacional Agencia Riobamba, through a situational diagnosis and comparative financial analysis in which the limited growth of financial intermediation in relation to local competition was established, within the same operating segment. The purpose of the study was to design financial strategies oriented to the growth and sustainability of the entity and therefore to the economic and social development of its clients through long-term financial relationships. In its development, various research techniques were applied, such as: a structured survey of clients in the Sierra Centro Zone, interviews with officials of the banking institution, as well as a diagnostic sheet applied to all employees of the Banco Internacional Riobamba offices. The population constitutes 1,357 clients corresponding to the different segments: executives, independent professionals, own business and companies. To determine the sample size, the proportional stratified sampling technique was used, obtaining a sample size of 300 surveys. The chi-square statistical test was applied in order to test the research hypothesis. It was concluded that the variables:
\end{abstract}

1 Escuela Superior Politécnica de Chimborazo, Facultad de Administración de Empresas, Riobamba, Ecuador, vicevallos@espoch.edu.ec

2 Escuela Superior Politécnica de Chimborazo, Facultad de Administración de Empresas, Riobamba, Ecuador, hernan.arellano@espoch.edu.ec

${ }^{3}$ Investigador Independiente, Riobamba, Ecuador, gsantillán@ gmail.com

${ }^{4}$ Escuela Superior Politécnica de Chimborazo, Facultad de Ciencias, Riobamba, Ecuador, paulina.valverde@espoch.edu.ec 
independent "Financial Strategies" and the dependent "Social Economic Growth", are related, demonstrating their reliability by calculating Cronbach's Alpha to the correlation data. In this way it was verified that "financial strategies do contribute significantly to social economic growth", regarding the case study.

Keywords: Financial Strategies, Economic Growth, Financial Intermediation, Corporate Social Responsibility.

\section{Resumen.}

La presente investigación se desarrolló en el Banco Internacional Agencia Riobamba, a través de un diagnóstico situacional y análisis financiero comparativo en el cual se estableció el limitado crecimiento de la intermediación financiera en relación a la competencia local, dentro del mismo segmento de operatividad. La finalidad del estudio fue diseñar estrategias financieras que aporten al crecimiento de la participación en el mercado de la entidad y aporten al desarrollo económico y social de sus clientes a través de relaciones financieras de largo plazo con los diversos segmentos actuales y potenciales. En su desarrollo se aplicaron diversas técnicas de investigación como: la encuesta estructurada a clientes de la Zona Sierra Centro, entrevistas a los funcionarios de la institución bancaria, así como una ficha de diagnóstico aplicada a todos los empleados del Banco Internacional oficinas Riobamba. La población constituyen 1.357 clientes correspondientes a los diferentes segmentos: ejecutivos, profesionales independientes, negocio propio y empresas. Para determinar el tamaño de la muestra se empleó la técnica del muestreo estratificado proporcional obteniendo un tamaño de la muestra de 300 encuestas. Se aplicó la prueba estadística chi-cuadrado, con la finalidad de comprobar la hipótesis de investigación. Pudo concluirse que las variables: independiente "Estrategias Financieras" y la dependiente "Crecimiento Económico Social", se encuentran relacionadas demostrándose su fiabilidad mediante el cálculo Alfa de Cronbach a los datos de correlación. De esta manera se comprobó que "las estrategias financieras si aportan significativamente al crecimiento económico social", respecto al caso de estudio.

Palabras claves: Estrategias Financieras, Crecimiento Económico, Intermediación Financiera, Responsabilidad Social Empresarial.

\section{Introducción.}

Las instituciones financieras del Ecuador se enfrentan a grandes retos que abarcan desde la necesidad de aumentar la eficiencia operativa que asegure el cumplimiento de reglamentaciones hasta el incremento de las ofertas de productos más inclusivos que puedan centrarse en el establecimiento de términos de plazos más largos y costos más bajos. (Estévez - Tórrez, 2019) 
Las entidades bancarias han experimentado importantes cambios debido al entorno económico altamente competitivo. La intermediación financiera es el servicio que se hace para contactar a los poseedores de recursos financieros (dinero, bienes de capital, captación de recursos, etc.) con aquellas personas físicas o jurídicas que necesitan dichos recursos financieros (préstamos), para utilizarlos y generar utilidades. (Escoto, 2011)

La gestión de las instituciones financieras contempla varios aspectos: las operaciones de intermediación, la administración de riesgos, la calidad de servicios y la responsabilidad social.

En las operaciones de intermediación bancaria se considera tanto colocaciones como captaciones de dinero. Las captaciones son los recursos del público que han sido realizados a través de depósitos a la vista, depósitos a largo plazos y otros. Las captaciones generan un costo financiero para los bancos (tasa pasiva). Las colocaciones es el mecanismo utilizado por el sistema financiero que permite poner en circulación el dinero que se obtiene de la captación del público, en otras palabras es la concesión de créditos a personas naturales o jurídicas que necesitan financiar sus actividades productivas o de consumo, los préstamos generan ingresos a través de los intereses y cargos admirativos para la institución financiera (tasa activa).

El banco Internacional S.A. está entre de los bancos más grandes del Ecuador, cuenta con agencias en 18 provincias del país y 88 oficinas a nivel nacional. Al hacer un análisis comparativo de la intermediación financiera de la agencia Riobamba frente a la competencia, se estableció un limitado crecimiento; en el año 2019 apenas se registra un 16\% de intermediación financiera en el mercado local, lo que implica mayores captaciones y baja colocación de créditos, por ende excesos de liquidez y baja rentabilidad. La pregunta de investigación que se propone resolver fue: ¿de qué manera aportan las estrategias financieras del Banco Internacional agencia Riobamba en el crecimiento económico social de sus clientes?

Frente al problema de intermediación financiera del banco Internacional agencia Riobamba, la investigación plantea un conjunto de estrategias para solucionar la baja colocación de créditos en relación a la captación de recursos y de esta forma dinamizar la cartera en todos los segmentos: ejecutivos altos y bajos, propietarios de pequeños negocios, profesionales en diferentes ramas y empresarios en general, si se mejora la atención crediticia para sus necesidades, también se logrará mejorar su calidad de vida, dinamizando el desarrollo económico social de la provincia y zona central del país.

El fin principal de la gestión financiera es procurar maximizar los beneficios empresariales; para lo cual será necesario contar con estrategias financieras que permitan conseguir los objetivos planteados. La administración de la entidad financiera tiene como prioridad mantener niveles adecuados de intermediación financiera en relación al promedio nacional, por considerarse la actividad primordial generadora de nuevos ingresos, por tanto es necesario una gestión prudente en el manejo de costos que asegure la eficiencia en sus operaciones. 


\section{¿Qué son las estrategias?}

El concepto de estrategia aplicado al ámbito empresarial, surge en los años sesenta, y ha ido evolucionando en función de los sistemas de dirección y los problemas internos y externos de la empresa, en este contexto se considera que las estrategias abarcan todas las actividades críticas de la empresa, dando un sentido común de unidad, dirección y propósito, así como facilitando los cambios necesarios en el entorno empresarial. (Rico, 2017, citado en Santillán 2018).

Según el autor Lobato, menciona que la estrategia empresarial busca la consecución de ventajas competitivas las cuales tienen que distinguirse de las de la competencia, para ello pondrá a disposición sus recursos y capacidades a fin de cumplir sus metas y objetivos en el mediano y largo plazo. (Lobato, 2015).

Los autores Kaplan y Norton establecen que las estrategias reflejan la manera en que la empresa genera valor para sus dueños y para los clientes, son la medida de actuación de la gestión empresarial. (Kaplan \& Norton, 2014)

Consecuentemente las estrategias parten de un diagnóstico de la entidad, de donde se obtiene las principales variables que se sintetizan en la matriz FODA (Fortalezas, Oportunidades Debilidades y Amenazas). Las Fortalezas y debilidades son internas y las Amenazas y Oportunidades son externas.

\section{Estrategias Financieras}

Las estrategias financieras son parte del proceso de planificación y gestión estratégica de una empresa, son las que orientan la obtención de recursos para financiar necesidades operativas de la empresa, como también generan las alternativas de inversión en función de los objetivos, de misión y visión empresarial. En este contexto las estrategias financieras procuran el uso eficiente de los recursos, analizando el menor costo posible de financiamiento, así como una capacidad de inversión y recuperación eficiente mediante la rotación de sus ventas.

La gestión eficiente de los recursos económicos incluye tres tipos de decisiones y por ende de estrategias financieras claves: en primer lugar la determinación de necesidades (inversión), la necesidad de obtención de fondos (financiamiento) y la necesidad de optimizar recursos (operación).

Uno de los objetivos fundamentales de las estrategias financieras es la rentabilidad de las inversiones mediante el análisis del circulante, combinando eficientemente liquidez y solvencia. También será necesario el análisis del riesgo crediticio, a fin de garantizar una adecuada rotación que permita cubrir y hacer frente a obligaciones de corto y largo plazo. Por ende las estrategias se clasifican en estrategias corto y de largo plazo Las estrategias de corto plazo procuran la optimización del capital de trabajo mediante la óptima administración del efectivo e inversiones 
temporales, gestión del crédito, control de inventarios y la selección de las mejores alternativas de financiamiento.

El financiamiento a corto plazo, está constituido por: fuentes espontaneas y créditos contratados; las fuentes espontaneas surgen del giro del negocio principalmente de los proveedores quienes contribuyen sobre todo con capital de trabajo y no generan costos de capital o intereses. En tanto que los créditos contratados son recursos negociados con instituciones financieras necesarias para cubrir déficits de flujos de caja por lo que generan costos financieros (intereses), en función del monto y plazos.

Las estrategias financieras de largo plazo están relacionadas con las inversiones en activos fijos o de larga duración que necesitan las empresas en empeño de ampliaciones de capacidad productiva, prestación de servicios, nuevas líneas de producción, cobertura de nuevos mercados, etc. En el largo plazo, las inversiones requieren de estudios de mayor alcance o de elaboración de proyectos de factibilidad, los cuales utilizan herramientas financieras que permiten medir los niveles de recuperación de las inversiones en términos monetarios (VAN), en términos de costos de oportunidad a través de la TIR, en función del tiempo (Período de recuperación); así mismo permiten un análisis de fuentes de financiamiento a través de tasas de descuento o Costo Promedio Ponderado del Capital.

\section{Crecimiento y Sostenibilidad}

El rol que juegan las entidades financieras es de suma importancia en el desarrollo económico social de un país, estimulan el crecimiento económico a través de la intermediación (Narváez Gómez, 2014)

El concepto de economía del desarrollo significa la capacidad que tiene la sociedad para crear riqueza, objetivo que es posible a través de la producción de bienes y/o servicios con las cuales satisfacen necesidades colectivas y generan mayor consumo, aportando a la calidad de vida de la población. Todo esto en un ámbito cada vez más competitivo.

La medida del valor de la actividad económica de un país se denomina Producto Interno Bruto (PIB), se calcula en base a la ponderación de la producción de los distintos sectores económicos en un período determinado. En el año 2017 el PIB sectorial bancario creció en un 10, 9\%. (EKOS, 2018)

La banca ecuatoriana en los últimos años atraviesa una etapa de importantes crecimiento y desarrollo, debido a la confianza generada luego de la crisis de los años 90 y las políticas de control y regulación que se reflejan básicamente en el crecimiento de depósitos y créditos. En el mes de agosto 2019 los depósitos totales registraron un crecimiento del 5,1\% respecto del año anterior, mientras que la cartera bruta en términos anules creció el 8.7\%. (ASOBANCA, 2019) 
De acuerdo a las estadísticas proporcionadas por el Banco central del Ecuador, la intermediación financiera ha tenido un crecimiento sostenido del 5,7\% en los últimos años. De esta manera el sistema financiero ecuatoriano es sólido y alejados de los factores que provocaron la crisis bancaria de 1.999. Los bancos actualmente manejan niveles de solvencia, liquidez y cobertura mucho más adecuados, lo que sin duda ha generado mayor confianza en la población. Estos aspectos han permitido que el sistema financiero cuente con la capacidad de incrementar depósitos y créditos (EKOS, 2018)

El desarrollo sostenible significa la satisfacción de necesidades de las presentes generaciones, sin llegar a comprometer en bienestar de las futuras. Esto significa la generación de conciencia colectiva para dar el uso adecuado a los limitados recursos naturales, recursos tangible e intangibles que dispone la sociedad, por lo cual se ha creado los objetivos de desarrollo sostenible a nivel mundial, son de aplicación universal y propenden disminuir los índices de pobreza, la protección del medio ambiente y buscar la prosperidad de la humanidad

\section{Responsabilidad Social Empresarial (RSE)}

La responsabilidad social es un tema de gran contenido social, cultural y económico, significa el compromiso de la población representadas en todo tipo de organizaciones públicas y privadas, para contribuir positivamente al incremento y creación del bienestar de la sociedad en general.

La Comisión de Seguimiento y Evaluación del dialogo social define a RSE como un conjunto de compromisos de diverso orden, económica, social y ambiental adoptados por las empresas, las organizaciones, instituciones públicas y privadas y que constituyen un valor añadido al cumplimiento de sus obligaciones legales, contribuyendo a la vez al progreso social y económico en el marco de un desarrollo sostenible. (Responsabilidad Social Corporativa, 2019)

Desde el punto de vista empresarial la RSE, constituye la manera en cómo sus actores sociales propietarios, administradores, funcionarios, etc., asumen el desenvolvimiento social, cultural y económico de una localidad, región o país. y el mundo, con la capacidad para escuchar a los intereses y contribuir con soluciones frente a sus clientes, el gobierno, prestadores de servicios, frente al medio ambiente en general.

La responsabilidad social empresarial (RSE) es una tendencia, es parte del modelo de gestión empresarial y constituye una alternativa de creación de valor, generando beneficios propios y beneficios sociales y colectivos. Contempla tres aspectos fundamentales: responsabilidad económica, social y ambiental.

Aplicado este concepto a la entidad bancaria significa que la misma, no solamente debe buscar su rentabilidad económica, tiene que ser básicamente una entidad comprometida con la generación de soluciones y alternativas, de apoyo permanente a nuevas iniciativas, de soporte a necesidades y por ende de aporte al desarrollo económico, social y cultural del país. 
En el aspecto cultural las entidades financieras tiene la responsabilidad de mejorar la cultura financiera de una población mediante la inclusión a segmentos tradicionalmente marginados, desatendidos, incorporándoles con la prestación de servicios y asistencia técnica para generar emprendimientos productivos, mejores prácticas sociales que eviten el financiamiento ilegal y eviten por ende costos sociales innecesarios.

Las instituciones financieras no obstante de ser uno de los segmentos económicos más regulados y controlados sin embargo aún no han desarrollado una filosofía acorde a los principios de la RSE, por tanto la responsabilidad social se convierte en una necesidad en toda institución bancaria es necesario que la sociedad civil representada por el cliente esté consiente que tiene la posibilidad de premiar o castigar el accionar de la entidad cuando ella se aparta de lo que se considera buenas prácticas empresariales. (Haza, 2010).

\section{Metodología}

\section{Tipo de investigación}

La presente investigación tiene un enfoque cuantitativo por cuanto utiliza información financiera y tiene un enfoque cualitativo por cuanto permite la interpretación de datos mediante la elaboración y aplicación de escalas de Likert. El nivel de investigación es carácter descriptivo por cuanto se limita a presentar las variables tal como se presentan en la realidad y en un momento específico. (Carrasco, 2015)

\section{Métodos de Investigación}

\section{Método Inductivo}

Partiendo de manifestaciones particulares y específicas describen los resultados de las observaciones y permiten hacer generalizaciones o enunciados universales.

\section{Método Analítico - Sintético}

En este caso permitió examinar la cartera de crédito local, con el propósito de conocer las causas de las variaciones en los procesos de intermediación financiera.

\section{Población y Muestra}

La población está constituida personas naturales y jurídicas correspondientes a los diversos segmentos de clientes con un total de 1.357. Para determinar el tamaño de la muestra se utilizó el muestreo estratificado, la proporción asignada a cada segmento constituye el porcentaje que representa cada segmento en relación al total. Mediante fórmula se estableció el tamaño de la muestra de 300 encuestas. 
TABLA 1. Población y Muestra

\begin{tabular}{lccc}
\hline \multicolumn{1}{c}{ TIPO DE CLIENTES } & NÜMERO & $\%$ & $\begin{array}{c}\text { MUESTRA ESTRATIFI- } \\
\text { CADA PROPORCIONAL }\end{array}$ \\
\hline Ejecutivos medio y altos & 320 & 23,58 & 71 \\
Profesionales Independientes & 378 & 27,86 & 84 \\
Negocio Propio & 571 & 42,08 & 126 \\
Empresas (pequeñas, medianas & 88 & 6,48 & 19 \\
y grandes) & & & $\mathbf{3 0 0}$ \\
TOTAL & $\mathbf{1 3 5 7}$ & $\mathbf{1 0 0 \%}$ & \\
\hline
\end{tabular}

Elaboración: Grupo de Investigación

\section{Técnicas de recolección de la información}

Se requirió de información de diversas fuentes, por lo que se utilizó técnicas de recolección tales como: entrevista a gerentes de la Zona 3, encuesta estructurada a los clientes y una ficha de diagnóstico aplicada a los empleados de la entidad.

\section{Resultados}

\section{Características del target}

Del total de encuestados, el $36.7 \%$ corresponde a clientes mujeres y el 63,3\% clientes hombres. Mayoritariamente los clientes son hombres; este dato es importante al momento de establecer el target para establecer o empaquetar propuestas de nuevos productos y servicios financieros.

El 53.3\% de encuestados se encuentra en los rangos comprendidos entre 31 años a 50 años de edad; este dato permite identificar aversión al riesgo.

\section{Resultados de la encuesta}

En primer lugar se procedió a determinar la escala de fiabilidad con las siguientes variables: calidad en la prestación de servicio, respuesta oportuna a requerimientos de información, asesoría de créditos, requisitos en préstamos, tasas, plazos y montos; así como incidencia en el crecimiento económico. 
Figura 1 Percepción de los clientes Banco Internacional Agencia Riobamba

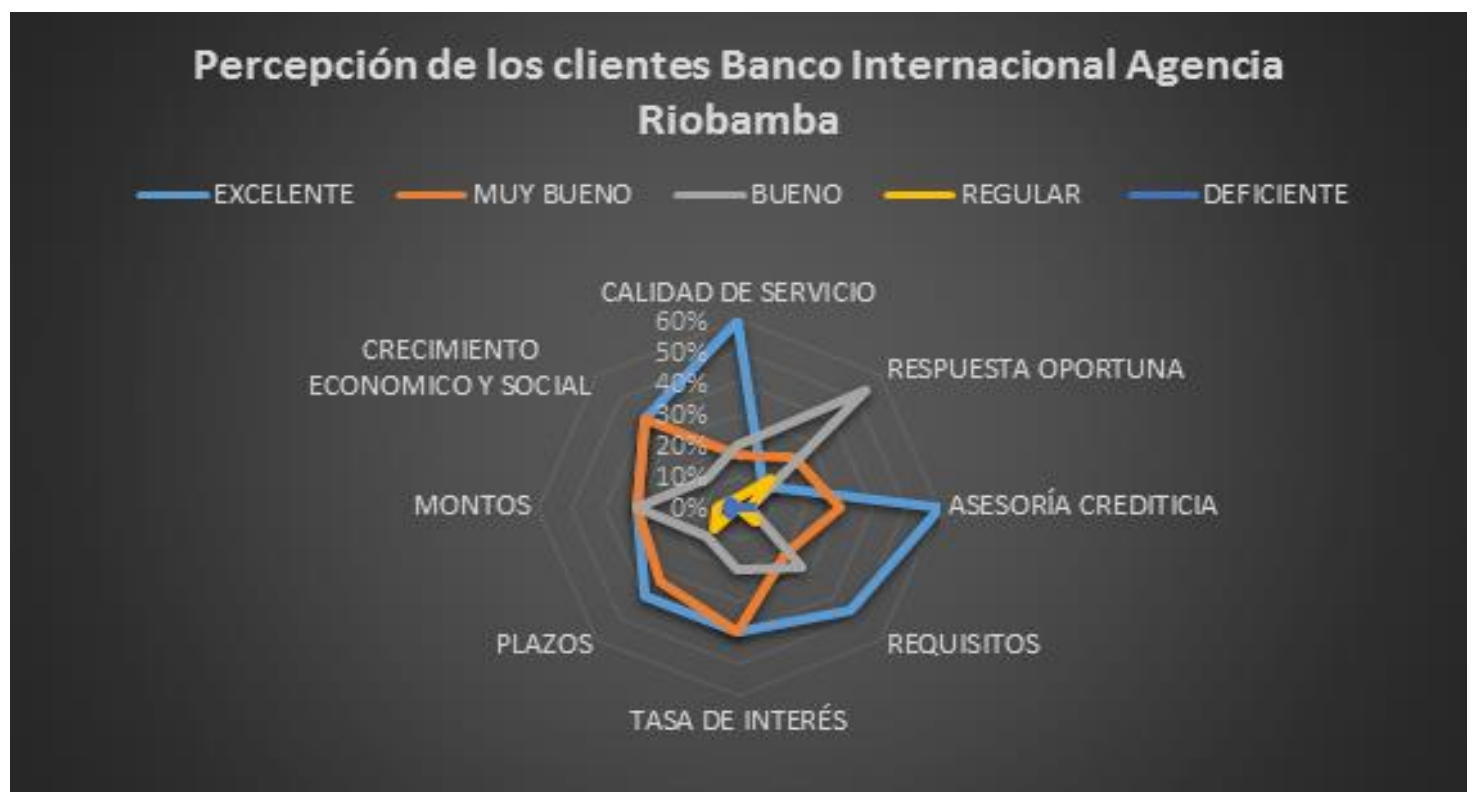

Elaboración: Grupo de Investigación

\section{Análisis de resultados}

Calidad de servicios.- el $60 \%$ de encuestados manifiesta que el servicio prestado por el banco Internacional en la agencia Riobamba es excelente, el 3,3\% considera que el servicio es regular. Las dos alternativas excelente y muy bueno suman el 76,7\% en satisfacción; sin embargo al comparar con el estándar de medición, establece un $90 \%$ como mínimo para considerarlo aceptable.

Respuesta oportuna a requerimientos.- en cuanto a la respuesta oportuna el 53,3\% lo califica como bueno; $23.3 \%$ muy bueno y el 13,3\% regular. Esta es una variable sensible que tiene que ser mejorada.

Asesoramiento en créditos.- El asesoramiento para la concesión de créditos fue calificada entre excelente y muy bueno con el 90\%; apenas el 3,3\% lo ha calificado como deficiente. Esta situación constituye una fortaleza para la entidad financiera.

Requisitos para acceder a créditos.- el 46,7\% de clientes opinan que los requisitos para acceder a los créditos es regular. Cabe resaltar que uno de los problemas principales es el incumplimiento en la justificación de ingresos, la informalidad no permite elevar el monto del crédito por la capacidad de pago. 
Tasas de interés.- las tasas de interés en el país son reguladas a través del Banco Central del Ecuador y varían de acuerdo al segmento y destino específico: comercial, de consumo, inmobiliario, productivo, vivienda de interés social, microcrédito., etc. consecuentemente los clientes están de acuerdo con su aplicación.

Plazos.- El 40\% consideran que los plazos establecidos son adecuados, mientras que el 3,3\%los considera deficientes.

Montos.- Un 90\% califica los montos de crédito en el rango de excelente a bueno, mientras que el 3,3\% como deficientes.

Crecimiento económico social.- El $80 \%$ de encuestados considera que el otorgamiento de créditos por parte de banco Internacional si ha contribuido a mejorar las condiciones económicas de los beneficiarios, por ende han contribuido a mejorar su calidad de vida.

\section{Comprobación de hipótesis}

Para determinar el modelo estadístico a ser aplicado en la comprobación de hipótesis, se estableció que el presente estudio se ubica en la categoría de pruebas "no paramétricas" considerando las siguientes premisas: los datos obtenidos corresponden a una sola muestra, se evalúan dos variables cualitativas, en base a una prueba de independencia.

Debido a estas razones, se seleccionó la prueba estadística del chi cuadrado, considerando para el mismo un nivel de significancia del 0,05 .

Sistema de Hipótesis.- las hipótesis de la presente investigación plantean un análisis de independencia entre las dos variables principales: estrategias financieras (independiente) y crecimiento económico social (dependiente). De tal manera, las hipótesis a ser comprobadas estadísticamente son las siguientes:

H0 = Las estrategias financieras no aportan significativamente al crecimiento y sostenibilidad de la agencia Banco Internacional en la ciudad de Riobamba.

H1 = Las estrategias financieras aportan significativamente al crecimiento y sostenibilidad de la agencia Banco Internacional en la ciudad de Riobamba.

Variable dependiente: Crecimiento y sostenibilidad

Variable independiente: Estrategias Financieras

Los indicadores considerados dentro de la variable independiente son los siguientes:

1. Calidad del servicio

2. Respuesta oportuna a requerimientos (financieros)

3. Asesoría Créditos (Asesoramiento Crediticio) 
4. Acceso a Créditos (Requisitos para acceso a créditos)

5. Tasa de interés (Créditos)

6. Plazo (Créditos

7. Monto (Créditos)

Una vez establecidos los ítems correspondientes a la variable independiente, se procedió a realizar tablas cruzadas con la variable dependiente (Crecimiento económico social). A continuación se presenta la tabla resumen de Prueba Chi Cuadrado para cada ítem:

Tabla 2. Resumen Prueba de Chi Cuadrado

\begin{tabular}{lcrrr}
\hline \multicolumn{1}{c}{$\begin{array}{c}\text { Variables de las tablas de contin- } \\
\text { gencia }\end{array}$} & $\begin{array}{c}\text { Valor Chi } \\
\text { Cuadrado } \\
\text { (Calculado } \\
\text { Spss) }\end{array}$ & G/L & $\begin{array}{c}\text { Nivel de Sig- } \\
\text { nificancia }\end{array}$ & $\begin{array}{r}\text { Valor Chi } \\
\text { Cuadrado } \\
\text { tabulado }\end{array}$ \\
\hline Calidad de Servicio*Crecimiento & 288.89 & 12 & 0.004 & 210,261 \\
\hline Respuesta Oportuna*Crecimiento & 362.5 & 12 & 0 & 210,261 \\
\hline Asesoría Crediticia* Crecimiento & 277.5 & 12 & 0 & 210,261 \\
\hline Acceso Crediticio* Crecimiento & 800 & 16 & 0 & 262,962 \\
\hline Tasa de Interés* Crecimiento & 168.75 & 8 & 0.031 & 155,073 \\
\hline Plazo* Crecimiento E.S & 665.67 & 16 & 0 & 262,962 \\
\hline Monto* Crecimiento E.S & 701.39 & 16 & 0 & 262,962 \\
\hline
\end{tabular}

Elaborado: Grupo de Investigación

En la Tabla 2 se consolidaron los resultados obtenidos de las variables del caso de estudio; luego de la comparación entre el valor calculado de Chi cuadrado, con los valores tabulados, se determinó que los valores calculados son mayores a los tabulados, por lo tanto se rechaza la hipótesis nula y se acepta la hipótesis alternativa. A continuación se muestra como ejemplo, la gráfica de la distribución de Chi - Cuadrado para la tabla de contingencia "Respuesta oportuna* Crecimiento Económico Social” 
Figura 1 Distribución Chi - Cuadrado

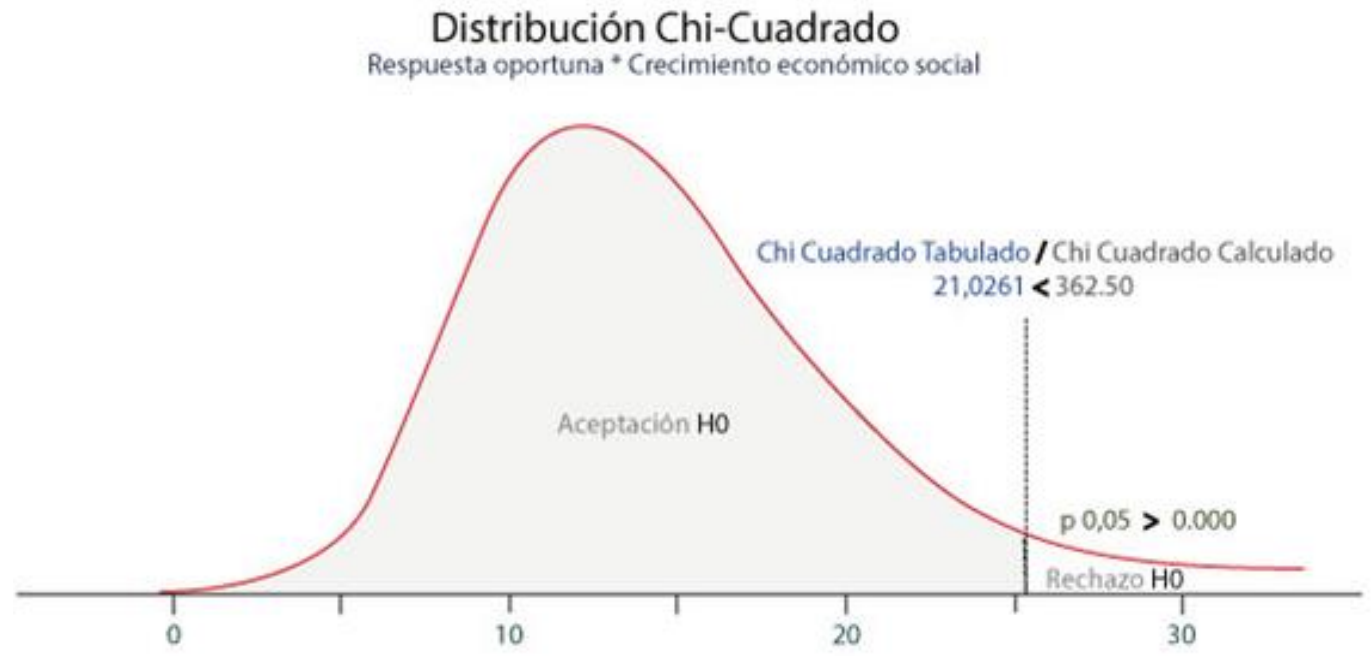

Fuente: Tomado de Trabajo de Titulación (Santillán, G, 2019)

Como se evidencia en la Figura 1. El valor de Chí - Cuadrado calculado (362.50) es mayor al tabulado (21.0261), ubicándose en la zona de rechazo de la hipótesis nula, razón por la cual se acepta la hipótesis alternativa (H1). El resto de variables mantienen el mismo comportamiento.

\section{Resultados del Análisis Situacional}

Para elaborar el análisis situacional, se aplicó el siguiente proceso:

1. Matriz FODA (Guía de entrevista y diagnóstico de evaluación)

2. FODA Jerarquizado:

Se utilizó la matriz de Holmes, para jerarquizar Fortalezas y Oportunidades y se utilizó el método de Ishikawa adaptado a una matriz llamada causa efecto para jerarquizar las Debilidades y Amenazas)

3. Matriz de Factores Estratégicos Priorizada:

Con la priorización de factores estratégicos, se establecieron 12 estrategias de tipo: ofensivas, defensivas, de reacción y de adaptación.

4. Matriz Estratégica FO FA DO DA

5. Nivel de impacto (Matriz estratégica de impacto) 
Tabla 3. Matriz Estratégica FO FA DO DA

\begin{tabular}{|c|c|c|}
\hline FACTORES EXTERNOS & OPORTUNIDADES & AMENAZAS \\
\hline$\square$ & $\begin{array}{l}04 \text { Aprobación de líneas de cré- } \\
\text { dito }\end{array}$ & $\begin{array}{l}\text { A2 Alto nivel de endeudamiento de } \\
\text { clientes }\end{array}$ \\
\hline FACTORES INTERNOS & $\begin{array}{l}01 \text { Capacidad de pago de clien- } \\
\text { tes }\end{array}$ & A3 Competencia desleal interna \\
\hline & $\begin{array}{l}02 \text { Utilización de plataforma tec- } \\
\text { nológica }\end{array}$ & $\begin{array}{l}\text { A4 Plataformas tecnológicas innova- } \\
\text { dora de la competencia }\end{array}$ \\
\hline FORTALEZAS & ESTRATEGIAS FO OFENSIVAS & ESTRATEGIAS FA REACCIÓN \\
\hline $\begin{array}{l}\text { F1 Servicio y atención a } \\
\text { clientes personalizada }\end{array}$ & $\begin{array}{l}\text { F1 } 04 \text { Calificar líneas de crédito } \\
\text { con presupuesto de inversión } \\
\text { por un año }\end{array}$ & $\begin{array}{l}\text { F1 A2 Crear un plan de subrogación } \\
\text { de deudas que incluyan capital de tra- } \\
\text { bajo e inversión }\end{array}$ \\
\hline $\begin{array}{l}\text { F2 Desarrollo de nuevas } \\
\text { iniciativas de negocios }\end{array}$ & $\begin{array}{l}\text { F2 } 01 \text { Desarrollar nuevas iniciati- } \\
\text { vas de negocios en función de la } \\
\text { capacidad de pago actual del } \\
\text { cliente }\end{array}$ & $\begin{array}{l}\text { F2 A3 Negociar la tasa de interés so- } \\
\text { bre discrecionalidad permitida de } \\
\text { acuerdo a plazo, monto y destino del } \\
\text { crédito }\end{array}$ \\
\hline $\begin{array}{l}\text { F8 Bases de clientes seg- } \\
\text { mentadas }\end{array}$ & $\begin{array}{l}\text { F8 } 02 \text { Incentivar a la base de } \\
\text { clientes objetivo en la utilización } \\
\text { de la banca on line. }\end{array}$ & $\begin{array}{l}\text { F7 A4 Desarrollar créditos a largo } \\
\text { plazo, sobre bases de clientes con re- } \\
\text { cord positivo de pagos o con garantías } \\
\text { constituidas }\end{array}$ \\
\hline
\end{tabular}

F7 Calificación de riesgo

AAA

DEBILIDADES ESTRATEGIAS DO ADAPTACIÓN ESTRATEGIAS DA DEFENSIVAS

D1 Elevada concentración en la concesión de crédi-

D1 01 Diversificar el portafolio

D1 A2 Ajustar la tabla de amortiza- de clientes ción de acuerdo a la actividad del tos cliente con pagos: mensual, trimestral, semestral o al término.

D5 Direccionamiento de visitas a clientes
D5 02 Elaborar un plan de seguimiento a la vinculación de clientes target por parte de los asesores de negocios y servicios
D5 A3 Aplicar tasas de interés acorde a la segmentación de cartera de créditos
D6 Cumplimiento presupuestario del crédito
D6 04 Realizar una proyección por clientes sobre productos financieros de acuerdo a su actividad
D6 A4 Utilizar herramientas tecnológicas para la aprobación de créditos en forma masiva

Elaboración: Grupo de Investigación 


\section{Nivel de impacto}

Seguidamente se procedió a determinar el nivel de impacto de cada estrategia, para lo cual se construyó una matriz de doble entrada: en las filas se ubicaron las estrategias y en las columnas las variables de impacto que la gerencia de las oficinas de Riobamba del banco Internacional coinciden y consideran significativas para priorizar el orden en la ejecución de las mismas. (Sangucho, 2011)

Las variables de impacto se establecieron acorde a la naturaleza de la institución (Imagen Corporativa, Rentabilidad, Clima Laboral, Costo de ejecución, Satisfacción del cliente) y aplicaron las siguientes ponderaciones:

- $\quad$ Impacto Nulo = 0

- $\quad$ Impacto Bajo = 1

- $\quad$ Impacto Medio = 3

- $\quad$ Impacto Alto = 5

- $\quad$ Impacto Muy Alto = 10

Tabla 4 Matriz estratégica de impacto

\begin{tabular}{lrrrrrr} 
ESTRATEGIAS & \multicolumn{7}{c}{\begin{tabular}{l} 
VARIABLES DE IMPACTO \\
\cline { 2 - 9 }
\end{tabular}} & $\begin{array}{l}\text { IMAGEN } \\
\text { CORPORA- } \\
\text { TIVA }\end{array}$ & $\begin{array}{c}\text { RENTABI- } \\
\text { LIDAD }\end{array}$ & $\begin{array}{c}\text { CLIMA } \\
\text { LABO- } \\
\text { RAL }\end{array}$ & COSTO & $\begin{array}{c}\text { SATISFACCIÓN } \\
\text { DEL CLIENTE }\end{array}$ & PROMEDIO \\
\hline $\begin{array}{l}\text { PONDERA- } \\
\text { CIÓN }\end{array}$ & $\mathbf{2 0 \%}$ & $\mathbf{3 0 \%}$ & $\mathbf{5} \%$ & $\mathbf{1 0 \%}$ & $\mathbf{3 5 \%}$ & $\mathbf{1 0 0 \%}$ \\
\hline E1 & 10 & 10 & 10 & 3 & 10 & $9.30 \%$ \\
\hline E2 & 10 & 0 & 5 & 0 & 10 & $5.75 \%$ \\
\hline E3 & 10 & 10 & 10 & 5 & 10 & $9.50 \%$ \\
\hline E4 & 10 & 10 & 3 & 3 & 10 & $8.95 \%$ \\
\hline E5 & 10 & 0 & 3 & 0 & 10 & $5.65 \%$ \\
\hline E6 & 5 & 3 & 1 & 1 & 10 & $5.55 \%$ \\
\hline E7 & 10 & 3 & 10 & 5 & 10 & $7.40 \%$ \\
\hline E8 & 3 & 3 & 0 & 0 & 10 & $5.00 \%$ \\
\hline E9 & 10 & 10 & 10 & 0 & 10 & $9.00 \%$ \\
\hline E10 & 3 & 1 & 1 & 1 & 10 & $4.55 \%$ \\
\hline E11 & 5 & 3 & 0 & 0 & 5 & $3.65 \%$ \\
\hline E12 & 10 & 10 & 3 & 0 & 10 & $8.65 \%$ \\
\hline
\end{tabular}

Elaboración: Grupo de Investigación 
Con las calificaciones obtenidas en la tabla 4 se procedió a establecer el orden de aplicación de las estrategias planteadas, como también a elaborar los planes operativos.

\section{Conclusiones.}

- Se ha demostrado estadísticamente mediante la prueba chi - cuadrado la hipótesis de investigación. Pudo concluirse que las variables: independiente "Estrategias Financieras" y la dependiente "Crecimiento y Sostenibilidad" se encuentran relacionadas demostrándose su fiabilidad mediante el cálculo del Alfa de Cronbach a los datos de correlación de chi cuadrado de Pearson. De esta manera se comprobó que las estrategias financieras sin aportan significativamente al crecimiento económico social, respecto al ámbito de acción de la entidad financiera.

- El análisis sobre la gestión financiera de captaciones y colocaciones en la plaza de Riobamba es muy dinámico en cuanto al crecimiento de productos a la vista y plazo, siendo un aporte importante en el desarrollo económico de la ciudadanía que confía sus recursos en la institución financiera. En la colocación de cartera de créditos el crecimiento de la curva es constante por lo que se demanda de estrategias financieras que permitan la motivación para que más personas y en mayores montos accedan al crédito en las mejores condiciones de atención y satisfacción de necesidades.

- Las estrategias financieras son una herramienta de gestión gerencial que permiten garantizar el cumplimiento de niveles de intermediación financiera en cada plaza donde el Banco Internacional mantiene presencia, se recomienda buscar siempre la mayor satisfacción de los clientes con un modelo de negocios de largo plazo no solamente circunstancial con enfoque hacia el mercado objetivo. Es esencial contar con productos financieros de calidad que sean competitivos, en un ámbito de transparencia, seguridad, eficiencia para general altos grados de satisfacción en los clientes internos y externos.

- Se evidencia la necesidad de gestionar el desarrollo y capacitación del talento humano, seguido de innovar los sistemas de información, realizar un seguimiento y control integral a través de sus líderes; así como perseguir el nivel de retención y vinculación de nuevos clientes a la bancarización que permita ampliar las soluciones financieras con eficiente y prudente administración de recursos económicos orientados a dinamizar la economía y desarrollo social de la localidad y el país.

- Las estrategias financieras diseñadas, jerarquizadas, priorizadas y valoradas de acuerdo a su impacto apoyarán al crecimiento en cantidad y calidad crediticia en la entidad financiera; las mismas están direccionadas a todos los sectores económicos de la ciudad: comercio, sector inmobiliario, consumo y microcrédito, en función de sus necesidades.

\section{Referencias Bibliográficas.}

Asobanca. (2019). Evolución de la banca privada ecuatoriana. https://www.google.com/search?client=firefox-b-d\&q=asobanca 
Carrasco, S. (2015). Metodología de la Investigación Científica. Pautas metodológicas para diseñar y elaborar el proyecto de investigación. Lima: Editorial San Marcos.

EKOS. (2018). Obtenido de https://www.ekosnegocios.com/articulo/evolucion-del-sistemafinanciero-algunos-indicadores

Escoto, R. (2011). Banca Comercial. Obtenido de Google: https://books.google.com.ec/books?id=oDIBV4vO54IC\&pg=PA32\&dq=intermediaci\%C 3\%B3n+financiera.+definici\%C3\%B3n\&hl=es\&sa=X\&ved=0ahUKEwj21_K9xrvoAhW mIbkGHa5JDgAQ6AEIJzAA\#v=onepage \&q=intermediaci\%C3\%B3n\%20financiera.\%2 Odefinici\%C3\%B3n\&f=false

Estévez - Torrez, Z. (2019). Problemas que afectan el desempeño del sistema financiero ecuatoriano en el siglo XXI. Obtenido de Eumed. net: https://www.eumed.net/rev/caribe/2019/04/sistema-financiero-ecuador.html

García, J., \& Hernández. (2014). Estrategias Financieras Empresariales. México: Grupo Editorial Patria.

Haza, B. (2010). Los bancos y la responsabilidad social como medición del éxito. Obtenido de http://revistas.pucp.edu.pe/index.php/derechopucp/article/view/2930

Kaplan, \& Norton, D. (2014). Mapas Estratégicos. Harvard Business Press.

Lobato, D. (2015). Estrategia de Empresas. Rio de Janeiro, Brasil: Editora FGV.

Madroño, M. (2016). Administración Financiera del Circulante. México: Publicaciones Instituto Mexicano de Contadores Públicos.

Narváez Gómez, G. (2014). Desarrollo del sector financiero como factor de crecimiento económico en Ecuador y América Latina . Quito: FLACSO.

Responsabilidad Social Corporativa. (2019). Google. Obtenido de Responsabilidad Social: Definición: $\quad \mathrm{http}: / /$ responsabilidad-social-corporativa.com/responsabilidad-socialempresarial-definicion/

Rico, M. (2017). Fundamentos Empresariales. Madrid: ESIG Editorial.

Sangucho, A. (2011). Planificación Estratégica. Quito, Pichincha, Ecuador.

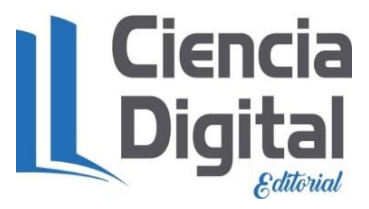




\section{PARA CITAR EL ARTÍCULO INDEXADO.}

Arellano Diaz, H. O., Cevallos Vique, V. O., Santillán Valle, G. M., \& Valverde Aguirre, P. E. (2020). Estrategias financieras para la sostenibilidad y el crecimiento del banco internacional agencia Riobamba período 2019 - $\quad$ 2021. Ciencia Digital, 4(2), $48-64$. https://doi.org/10.33262/cienciadigital.v4i2.1196

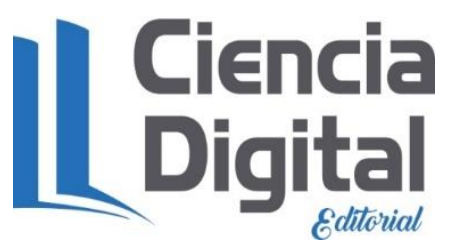

El artículo que se publica es de exclusiva responsabilidad de los autores y no necesariamente reflejan el pensamiento de la Revista Ciencia Digital.

El artículo queda en propiedad de la revista y, por tanto, su publicación parcial y/o total en otro medio tiene que ser autorizado por el director de la Revista Ciencia Digital.
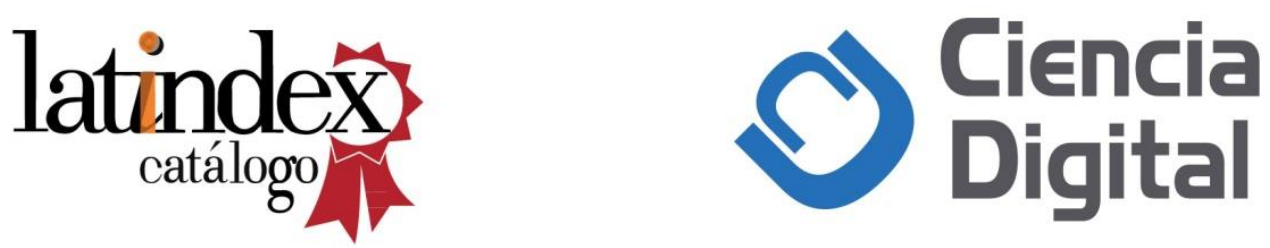